\title{
Tidal disruption events seen in the XMM-Newton slew survey
}

\author{
Richard Saxton ${ }^{1}$, S. Komossa ${ }^{2}$, Andrew Read ${ }^{3}$, Paulina Lira ${ }^{4}$, \\ Kate D. Alexander ${ }^{5}$ and Iain Steele ${ }^{6}$ \\ ${ }^{1}$ XMM SOC, ESAC, Apartado 78, 28691 Villanueva de la Cañada, Madrid, Spain \\ email: rsaxton@sciops.esa.int \\ ${ }^{2}$ QianNan Normal University for Nationalities, Longshan Street, Duyun City of Guizhou \\ Province, China \\ ${ }^{3}$ Dept. of Physics and Astronomy, University of Leicester, Leicester LE1 7RH, U.K. \\ ${ }^{4}$ Universidad de Chile, Observatorio Astronomico Nacional Cerro Calan, Santiago, Chile \\ ${ }^{5}$ Harvard-Smithsonian Center for Astrophysics, 60 Garden St., Cambridge, MA 02138, USA \\ ${ }^{6}$ Astrophysics Research Institute, Liverpool John Moores University, Liverpool CH41 1LD, UK

\begin{abstract}
XMM-Newton performs a survey of the sky in the $0.2-12 \mathrm{keV}$ X-ray band while slewing between observation targets. The sensitivity in the soft X-ray band is comparable with that of the ROSAT all-sky survey, allowing bright transients to be identified in near real-time by a comparison of the flux in both surveys. Several of the soft X-ray flares are coincident with galaxy nuclei and five of these have been interpreted as candidate tidal disruption events (TDE). The first three discovered had a soft X-ray spectrum, consistent with the classical model of TDE, where radiation is released during the accretion phase by thermal processes. The remaining two have an additional hard, power-law component, which in only one case was accompanied by radio emission. Overall the flares decay with the classical index of $t^{-5 / 3}$ but vary greatly in the early phase.
\end{abstract}

Keywords. X-rays: galaxies, galaxies: active

\section{Introduction}

A tidal disruption event (TDE) occurs when a stellar object is gravitationally destroyed and subsequently accreted by a super-massive black hole (SMBH). It represents the onset of a brief phase of accretion activity in an otherwise dormant galactic nucleus and is characterised by a sudden dramatic rise in luminosity, and a decline to quiescence on a timescale of months to years.

Early work predicted that stellar debris would return into a tightly bound orbit about the SMBH and form an optically thick accretion structure close to the SMBH (e.g. Rees 1988). This would emit thermal radiation of temperature $k T_{\text {eff }} \approx 40 M_{6}^{-1 / 4} \mathrm{eV}$ (Ulmer 1999) whose high energy tail would be visible in the soft X-ray band. Pioneering observations by ROSAT identified several X-ray flares in non-active galaxies (Komossa \& Bade 1999; see Komossa 2015 for a recent review) characterised by a very soft X-ray spectrum. Explanations such as variable AGN, blazars, ULX, supernova or supernova remnants were excluded by the amplitude of the variability (factor $>1000$ for NGC 5905 and RXJ 1242-1119; Komossa et al. 2004) and high luminosities of up to several $\times 10^{44}$ ergs/s. As their light curves have fallen consistently with the predicted rate, $t^{-5 / 3}$ (Rees 1988, Guillochon \& Ramirez-Ruiz 2013), TDEs are the most likely explanation for these events. 


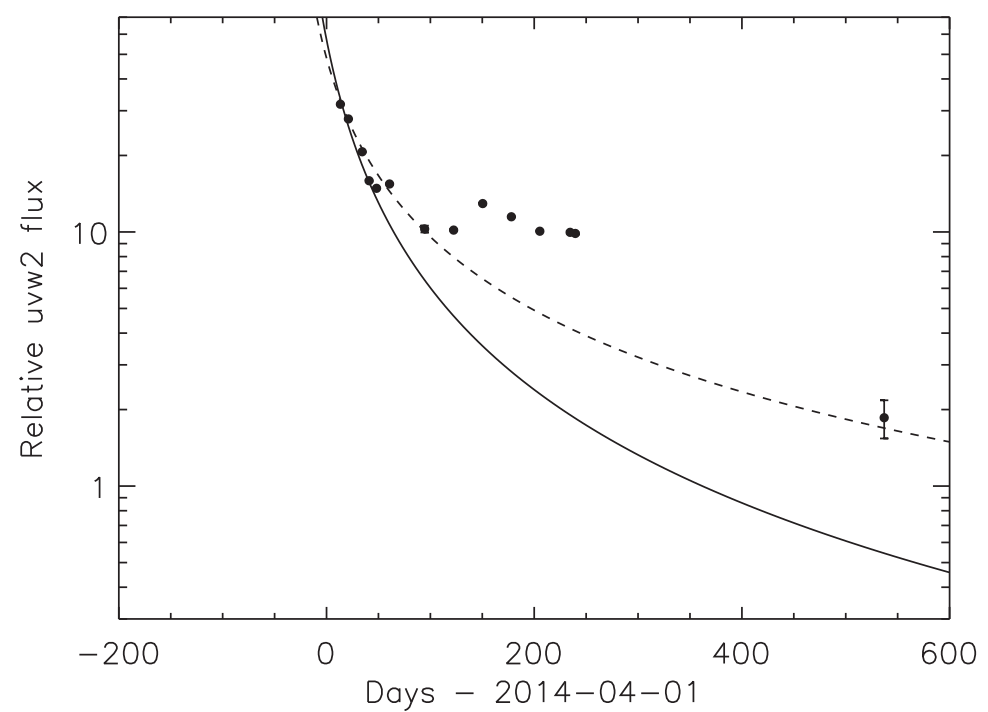

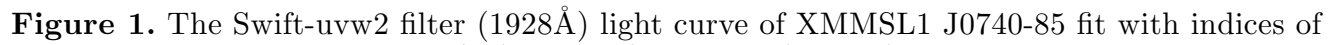
$-5 / 3$ (solid line) and -1.2 (dashed).

\section{XMM-Newton slew survey}

The XMM-Newton slew survey collates the data taken by the EPIC-pn camera while the satellite moves between targets. It has a sensitivity in the $0.2-2 \mathrm{keV}$ energy band which is well matched with the ROSAT all-sky survey. Comparisons between these two surveys have led to the detection of several transient sources, including five candidate TDEs (Tab. 1). NGC 3599 and SDSS J1323+48 were found in archival data (Esquej et al. 2008) while SDSS J1201+30 (Saxton et al. 2012), 2MASX J0619-65 (Saxton et al. 2014) and XMMSL1 J0740-85 (Saxton et al. 2017) were found at the peak of their emission and had their decays monitored with Swift and XMM-Newton. XMMSL1 J063045.9-603110, a bright slew transient from 2011, has been proposed as a TDE in a dwarf galaxy (Mainetti et al. 2016). However, a contemporary optical spectrum localised the flare to the local group (B. Cenko, p.comm.) and so this remains unconfirmed.

The initial estimate of the tidal disruption rate from the XMM-Newton slew data was given as $2.3 \times 10^{-4} \mathrm{gal}^{-1} \mathrm{yr}^{-1}$ (Esquej et al. 2008) based on two TDE. Scaling this calculation for the five which have now been found in data taken up until the end of 2014, gives a somewhat lower value of $1.4 \times 10^{-4} \mathrm{gal}^{-1} \mathrm{yr}^{-1}$.

\subsection{Temporal characteristics}

The XMM-Newton slew survey TDEs have faded in X-rays over periods of 6 months and more with an index compatible with $t^{-5 / 3}$ in line with the ROSAT TDE and the original expectations. In XMMSL1 J0740-85 the UV flux fell with a flatter index of $\sim 1.2$ (Fig. 1). This could be due to more flux entering the UV band as the flare cools. In detail though, the UV curve is seen to recover after about 100 days; a behaviour seen in other TDE (e.g. LeLoudas et al. 2016.). In analogy with the "supernova hump" in GRB this may imply a secondary emission mechanism; possibly circularisation and infall (LeLoudas et al. 2016; Krolik et al. 2016) or expelled unbound debris shocking against the ambient medium (Khokhlov \& Melia 1996).

There is a lot of variability in the X-ray light curves. About 20 days after discovery, SDSS J1201+30 dropped in X-ray flux by a factor 50 within 1 week (Fig. 2). A similar 


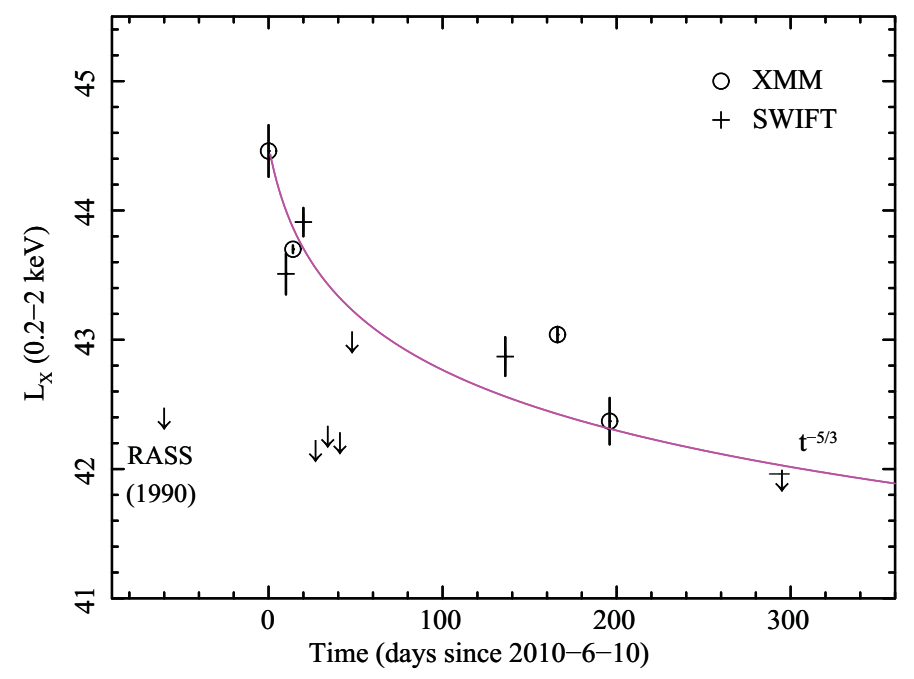

Figure 2. The $0.2-10 \mathrm{keV}$ light curve of SDSS J1201+30 fit.

drop after $\sim 30$ days was seen in XMMSL1 0740-85. The spectrum actually softens during the drop in 0740-85 excluding variations in neutral absorption and leaving ionised absorption or a change in the intrinsic emission as possible causes. Liu, Li \& Komossa (2014) interpret the dip as evidence for a close binary black hole system, where the fall back of material onto the primary black hole is interrupted by the presence of the secondary.

Simulations have shown that the development of a TDE light curve is dependent on when the streams of tidal debris intersect each other (Hyashaki et al. 2013; Guillochon \& Ramirez-Ruiz 2015; Shiokawa et al. 2015). Early interactions appear to be rare and in the majority of cases circularisation occurs late and at a large distance from the SMBH, leading to a delayed TDE with a longer rise to peak and a plateau that lasts months or even years. NGC 3599 with a plateau of $\geqslant 18$ months may have been a delayed TDE (Saxton et al. 2015). Optically-selected TDEs have either no X-ray emission or flat X-ray light curves with $L_{X}$ of a few $\times 10^{41-42} \mathrm{ergs} / \mathrm{s}$ (e.g. ASSASN 14li, Holohein et al. 2016; ASSASN 15lh, Margutti et al. 2016). We are investigating a TDE recently found in the slew survey with similar behaviour. It could be that these TDEs are all delayed in some way, however there is a similarity between the luminosity and X-ray light curves of these events and those of extremely luminous young supernova remnants such as SN 2006jd (Stritzinger et al. 2012) and SN 2010jl (Chandra et al. 2012). The featureless TDE spectra, nevertheless, argue against the X-ray emission being produced by ISM shocks.

\subsection{Spectral characteristics}

Material falling back to the inner orbits around a $10^{6-7} M_{\odot}$ black hole will heat up and emit radiation with an equivalent temperature of a few tens of $\mathrm{eV}$. While this broadly matches the emission seen in the soft X-ray spectrum in no case have we been able to join the X-ray and UV emission with a single thermal multi-colour disk model.

In two of the slew sources, XMMSL1 J0740-85 and 2MASX J0619-65, a hard component is visible, stretching out to at least $10 \mathrm{keV}$. A similar component was seen in NGC 4845 (Nikolajuk \& Walter 2013) and ASASSN-15oi (Holoien et al. 2016b). Weak radio emission was seen in NGC 4845 and XMMSL1 J0740-85 (Alexander et al.2016) consistent with an outflow or a weak relativistic jet. This implies that a source of high-energy comptonising 
Table 1. Candidate TDE found in the XMM-Newton slew survey.

\begin{tabular}{|c|c|c|c|c|c|}
\hline \multirow[t]{2}{*}{ Name } & \multirow[t]{2}{*}{$\mathrm{z}$} & \multirow[t]{2}{*}{$\log \left(L_{b \circ l}\right)$} & \multicolumn{3}{|c|}{ Flare $?^{1}$} \\
\hline & & & Soft-X & | Hard-X & | Radio \\
\hline NGC 3599 & 0.002 & 42 & Y & $\mathrm{N}$ & $?$ \\
\hline SDSS $1323+48$ & 0.07 & 44 & $\mathrm{Y}$ & $\mathrm{N}$ & ? \\
\hline SDSS $1201+30$ & 0.146 & 45 & $\mathrm{Y}$ & $\mathrm{N}$ & $\mathrm{N}$ \\
\hline 2MASX 0619-65 & 0.07 & 44 & $\mathrm{Y}$ & $\mathrm{Y}$ & $\mathrm{N}$ \\
\hline XMMSL1 $0740-85$ & 0.019 & 44 & Y & $\mathrm{N}$ & Y \\
\hline
\end{tabular}

Notes:

${ }^{1}$ Was there a flare at this wavelength?

electrons, analogous to the comptonising corona of a steady-state AGN, can be produced during disruptions.

\subsection{Host galaxy}

A disproportionate fraction of optically-selected TDE are found in post-starburst galaxies (Arcavi et al. 2014), a feature that is shared by XMMSL1 J0740-85. Arcavi et al. (2014) suggested that starbursts triggered by galaxy mergers could produce binary black holes that dynamically enhance the TDE rate. Alternatively, starbursts could create central stellar overdensities where enhanced encounter (two body relaxation) rates lead to more TDE flares (Stone \& Metzger 2016).

\section{References}

Alexander, K. D., et al. 2016, arXiv:1610.03861

Chandra, P., et al. 2012, ApJ Letters,750, L2

Esquej, P., Saxton, R., Komossa, S., Read, A., \& Freyberg, M. J., 2008, A\&A, 489, 543

Guillochon, J. \& Ramirez-Ruiz, E. 2013, ApJ, 767, 25

Guillochon, J. \& Ramirez-Ruiz, E. 2015, ApJ, 809, 166

Hayasaki, K., Stone, N., \& Loeb, A. 2013, MNRAS, 434, 909

Holoien, T., Kochanek, C., Prieto, J., Stanek, K., Dong, S., et al. 2016, MNRAS, 455, 2918

Holoien, T., Kochanek, C., Prieto, J., Grupe, D., Chen, P., et al. 2016, arXiv:1602.01088

Khokhlov, A. \& Melia, F. 1996, ApJ 457, L61

Komossa, S. \& Bade, N. 1999, A\&A, 343, 775

Komossa, S., Halpern, J., Schartel, N., et al. 2004, ApJ, 603, L17

Komossa, S. 2015, JHEAp 7, 148

Krolik, J., et al., 2016, ApJ, 827, 127

Leloudas, G., Fraser, M., Stone, N., van Velzen, S., Jonker, P. G., et al. 2016, arXiv:160902927

Liu, F., Li, S., \& Komossa, K. 2014, ApJ, 786, 103

Mainetti, D, Campana, S. \& Colpi, M. 2016, A\& A, 592, 41

Margutti, R., et al. 2016, arXiv:1610.01632

Nikolajuk, M. \& Walter, R., 2013, A\& A, 552, 75

Rees, M. 1988, Nature, 333, 523

Saxton, R., Read, A., Esquej, P., et al. 2012, A\& A, 541, 106

Saxton, R., Read, A., Komossa, S., et al. 2014, A $\mathscr{E} A$, 572, 1

Saxton, R., et al. 2015, MNRAS, 454, 2798

Saxton, R., Read, A., Komossa, S., et al. 2017, A $₫ A$, 598, A29

Shiokawa, H., Krolik, J., Cheng, R., Piran, T., \& Noble, S. 2015, ApJ, 804, 85

Stone, N. \& Metzger, B. 2016, MNRAS, 455, 859

Stritzinger, M, Taddia, F. Fransson, C., et al. 2012, ApJ, 756, 173

Ulmer, A. 1999, ApJ, 514, 180 